\title{
New Cooked Fish Paste from Frozen Alaska Pollack Surimi
}

\author{
Eiji Nrwa, Kazuhaya Nishimura and Satoshi KanOH
}

Faculty of Fisheries, Mie University. Tsu 514, Japan

Received July 21, 1989

\begin{abstract}
A viscous fish-flesh sol, shio-surimi, prepared from frozen Alaska pollack surimi, water, sodium chloride and starch was cooked at $80^{\circ} \mathrm{C}$ for $30 \mathrm{~min}$. The cooked product remained viscous if the starch had been pregelatinized. The most viscous type of paste with a consistency of mayonnaise or tomato ketchup was obtained from the shio-surimi containing $4.5-9 \%$ of pregelatinized tapioca starch or potato starch and 150 or $300 \%$ of water. The viscosity of the paste was increased when salad oil or butter was further added to the shio-surimi. Furthermore, a type of paste with the consistency of French dressing was obtained by grinding the resulting paste with rice vinegar and salad oil or butter. Cooked fish pastes prepared in this way may be utilized as a new form of marine food.
\end{abstract}

Viscous salted fish-flesh paste, shio-surimi, is changed to an elastic gel, Kamaboko, on heating. In this case, the elasticity of the kamaboko is strengthened by the addition of raw starch, but is scarcely strengthened if the starch has been pregelatinized. ${ }^{1 /}$ In this paper, we deal with a new cooked fish paste with a consistency of mayonnaise or tomato ketchup that is obtainable from frozen Alaska pollack suri$\mathrm{mi}$, if an adequate amount of pregelatinized potato or tapioca starch and water have been added, the resulting paste being miscible with rice vinegar and salad oil or butter. These pastes may be utilized as a new form of marine food.

\section{Materials and Methods}

Materials. Non-salted frozen surimi of Alaska pollack, Theragra charcogramma (Taiyo Gyogyo Co., SA-grade) was used as the main starting material for the cooked fish paste. Raw and pregelatinized starches from corn, potato and tapioca were donated by San-ei Tohka Co. There were two types of the respective pregelatinized starches, one being dehydrated by a drum drier after pregelatinization, while the other was immediately pregelatinized by passing through an extruder.

Preparation of the cooked fish paste. The frozen surimi was thawed by leaving overnight in a refrigerator at $4{ }^{\circ} \mathrm{C}$ minced and then ground in an automatic mortar (Nitto Kagaku Co., ANM-200) with sodium chloride, water and starch at $4^{\circ} \mathrm{C}$ for $15 \mathrm{~min}$ to form the shio-surimi. The final concentration of sodium chloride was held at $3 \%$ of the water contained within the shio-surimi (the water content of the frozen surimi was $78 \%$ ). In the case of salad oil (Nisshin Seiyu Co., Nisshin Salad Oil) or butter (Yukijirushi Nyugyo Co., Snow Brand Hokkaido Butter) being added to the shio-surimi, the mixture was repeatedly homogenized for 30-60 sec five times in a homoblender (Sakuma Seisakusho Co., No. 500C) instead of grinding in the mortar. The viscous shio-surimi was packed into casings (Kureha Kagaku Kogyo Co., Krehalon casing, $2 \mathrm{~cm}$ diameter) and cooked at $80^{\circ} \mathrm{C}$ for $30 \mathrm{~min}$ in a water bath after closing both the ends of the casing. The resulting cooked fish paste was stored overnight in a refrigerator.

Visco-elasticity measurements. The dynamic viscoelasticity measurement of the cooked fish paste was carried out by using a dynamic rheometer (Toyoseiki Co., Rheolograph Sol, No. 653) on a sample prepared within the measuring cell. The shio-surimi was packed into the cell $\left(4 \times 1.5 \times 0.2 \mathrm{~cm}^{3}\right)$ and fixed to the rheometer together with a blade $\left(2.5 \times 1 \times 0.05 \mathrm{~cm}^{3}\right)$, and then heated by a thermo-module at $80^{\circ} \mathrm{C}$ for $30 \mathrm{~min}$. After lowering the temperature to $20^{\circ} \mathrm{C}$, the storage modulus $\left(G^{\prime}\right)$ and loss modulus $\left(G^{\prime \prime}\right)$ were recorded while vibrating the cell at a frequency of $3 \mathrm{~Hz}$ under a strain of $1 \%$

The viscosity measurement was carried out by using a rotatioal viscometer (Tokyo Keiki Co., BM type; cylindrical rotor: length, $3 \mathrm{~cm}$; diameter, $3.2 \mathrm{~mm}$; rotational speed, $6 \mathrm{rpm})$. Thirty mililiters of the cooked fish paste was poured into a beaker (diameter, $3 \mathrm{~cm}$ ), covered with small amount of liquid paraffin to prevent the evaporation of water during heating, and immersed for $30 \mathrm{~min}$ in a water bath previously adjusted to the prescribed temperature. The viscosity was read when the indication of the viscom- 
eter became constant after revolving the rotor (about $3 \mathrm{~min}$ ).

Photographs were taken of the particularly characteristic cooked fish paste.

\section{Results and Discussion}

The texture of the cooked fish paste was varied not only by the amount of water added but also by the kind of starch added. When raw starch was added, the paste felt elastic like kamaboko, but when the pregelatinized starch was added, the elasticity was weakened in most cases by increasing its amount, and the prcduct remained viscous when an adequate amount of water was added. If no pregelatinized starch was added to the shio-surimi containing a large amount of water, the resulting cooked fish paste was very unstable, and a large amount of water was separated during cooking.

Figure 1 shows the effect of the pregelatinized starch treated with the drum drier on the storage modulus $\left(G^{\prime}\right)$ and loss modulus $\left(G^{\prime \prime}\right)$ of the cooked fish paste at $20^{\circ} \mathrm{C}$. By increasing the amount of water added, $G^{\prime}$ and $G^{\prime \prime}$ were remarkably decreased. (The percentage of wa-

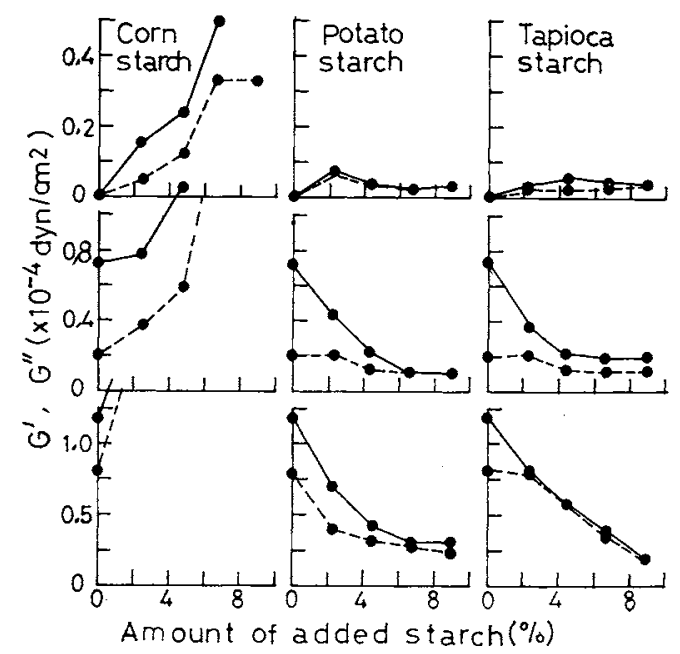

Fig. 1. Effect of the Pregelatinized Starch Treated by the Drum Drier on the Storage Modulus $\left(G^{\prime}\right)$ and Loss Modulus $\left(G^{\prime \prime}\right)$ of the Cooked Fish Paste at $20^{\circ} \mathrm{C}$.

- $G^{\prime} ;----G^{\prime \prime}$.

Amount of water added to the frozen surimi: $300 \%$ (top row); $150 \%$ (middle row); $100 \%$ (bottom row). ter, starch, salad oil, butter and rice vinegar added is expressed by the weight ratio to the frozen Alaska pollack surimi used as the main starting material according to the usual custom of the surimi-product industry). Irrespective of the amount of water added, both the moduli were remarkably increased by increasing the amount of corn starch added. For the cooked fish paste supplemented with $300 \%$ of water, the moduli were scarcely affected by the addition of the potato or tapioca starch, but for the paste supplemented with 100 or $150 \%$ of water, the moduli were decreased by increasing the amount of starch added. All the cooked fish pastes supplemented with $100 \%$ of water were in an unstable gel form while those supplemented with 150 or $300 \%$ of water were sols.

Figure 2 shows the effect of the pregelatinized starch treated by the extruder on $G^{\prime}$ and $G^{\prime \prime}$ of the cooked fish paste at $20^{\circ} \mathrm{C}$. Differing from the results in Fig. 1, for the cooked fish pastes supplemented with $300 \%$ of water, both the moduli were slightly increased by the addition of each starch, and for the pastes supplemented with 100 or $150 \%$ of water, they

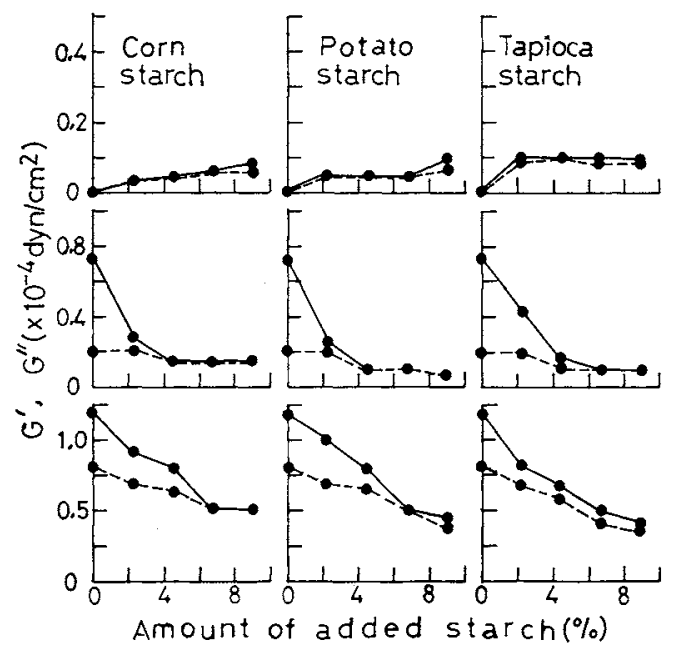

Fig. 2. Effect of the Pregelatinized Starch Treated by the Extruder on the Storage Modulus $\left(G^{\prime}\right)$ and Loss Modulus $\left(G^{\prime \prime}\right)$ of the Cooked Fish Paste at $20^{\circ} \mathrm{C}$.

-O- $G^{\prime}$; --- ---, $G^{\prime \prime}$.

Amount of water added to the frozen surimi: $300 \%$ (top row); $150 \%$ (middle row); $100 \%$ (bottom row). 


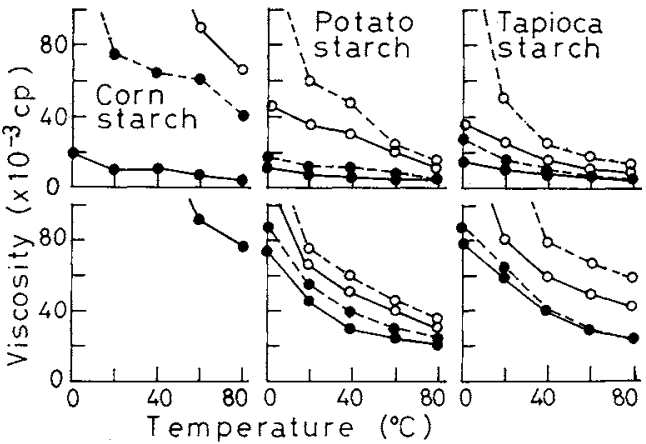

Fig. 3. Effect of Temperature on the Viscosity of the Cooked Fish Paste Supplemented with Various Amounts of Pregelatinized Starch Treated by Drum Drier.

Amount of water added to the surimi: $300 \%$ (upper row); $150 \%$ (lower row).

Amount of pregelatinized starch added: - $-2.25 \%$;

,$---- 4.5 \% ;-\bigcirc-, 6.75 \% ;--\bigcirc--, 9 \%$

were decreased by increasing the amount of corn starch added. Nearly the same results were obtained as those in Fig. 1 for the pastes supplemented with 100 or $150 \%$ of water and the potato or tapioca starch. The cooked pastes supplemented with $100 \%$ of water were unstable gels, but those supplemented with 150 or $300 \%$ of water were sols. Velvety smoothness was sensed for the cooked fish pastes supplemented with the potato or tapioca starch, but rough to the tongue was sensed for the paste supplemented with the corn starch.

Figure 3 shows the effect of temperature on the viscosity of the cooked fish paste supplemented with various amounts of pregelatinized starch treated with the drum drier. For all the cooked fish pastes, the viscosity was decreased by raising the temperature, but increased by increasing the amount of starch added. This increment was the most marked when the corn starch was added.

Figure 4 shows the same effect on the viscosity of the cooked fish paste supplemented with the pregelatinized starch treated by the extruder. A decrease in the viscosity with temperature, and its increase upon the addition of the starch showed nearly the same tendencies as those in Fig. 3, although the increase in viscosity was not as high as that from the addition of starch treated by the drum

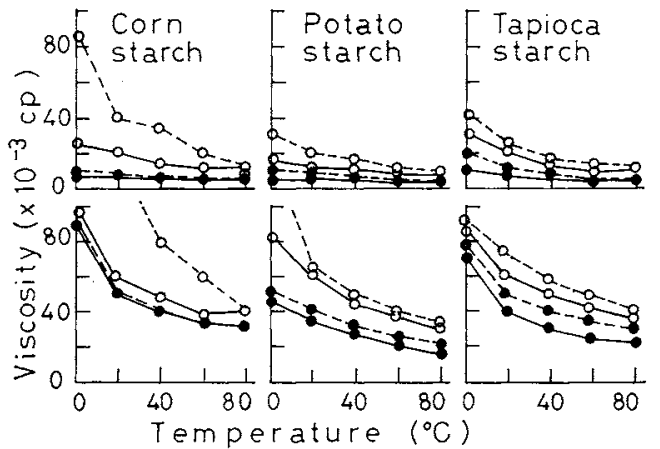

Fig. 4. Effect of Temperature on the Viscosity of the Cooked Fish Paste Supplemented with Various Amounts of Pregelatinized Starch Treated by the Extruder.

Amount of water added to the frozen surimi: $300 \%$ (upper row); $150 \%$ (lower row).

Amount of pregelatinized starch added: - - $2.25 \%$; ,$---- 4.5 \% ;-\mathrm{O}_{-}, 6.75 \% ;--\mathrm{O}^{--}, 9 \%$.

drier. For a commercial mayonnaise (Kewpie Co., Kewpie Mayonnaise), $G^{\prime}$ and $G^{\prime \prime}$ were $1.0 \times 10^{3}$ and $8.8 \times 10^{2} \mathrm{dyn} / \mathrm{cm}^{2}$ at $20^{\circ} \mathrm{C}$, respectively, and its viscosity was $5.4 \times 10^{4} \mathrm{cP}$ at this temperature. Judging from the value of $G^{\prime}$, $G^{\prime \prime}$ and viscosity, the cooked fish pastes supplemented with $150 \%$ of water and more than $4.5 \%$ of the potato or tapioca starch treated with the drum drier or extruder somewhat resembled the mayonnaise in consistency. Furthermore, for a commercial tomato ketchup (Kagome Co., Kagome Tomato Ketchup), $G^{\prime}$ and $G^{\prime \prime}$ were $2.6 \times 10^{2}$ and $4.8 \times 10^{2} \mathrm{dyn} /$ $\mathrm{cm}^{2}$ at $20^{\circ} \mathrm{C}$, respectively, and its viscosity was $2.8 \times 10^{4} \mathrm{cP}$ at this temperature. Therefore, the cooked fish pastes supplemented with $300 \%$ of water and more than $4.5 \%$ of the potato or tapioca starch treated with the drum drier somewhat resembled the tomato ketchup in consistency. In fact, these pastes also closely resembled the mayonnaise and tomato ketchup, respectively, from an organoleptic judgement. It was hard, however, to discriminate between the pastes supplemented with the potato starch and those with the tapioca starch, or to detect a textural difference between the pastes with the same species of added starches pregelatinized by different methods.

Figure 5 shows the effect of temperature on 


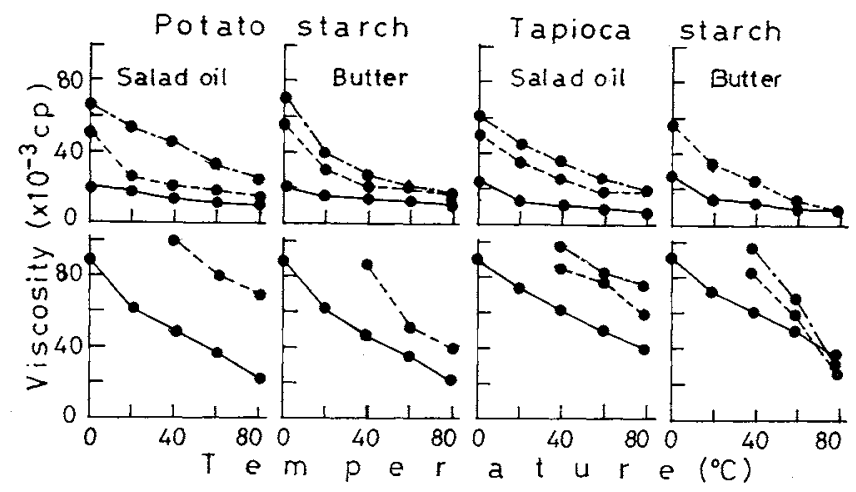

Fig. 5. Effect of Temperature on the Viscosity of the Cooked Fish Paste Supplemented with Salad Oil or Butter, Except for the $4.5 \%$ Pregelatinized Potato or Tapioca Starch Treated by the Drug Drier.

Amount of water added to the surimi: $300 \%$ (upper row); $150 \%$ (lower row).

Amount of salad oil or butter added: -,$- 0 \% ;--\mathbf{O}_{--}, 10 \% ;-\cdot-\mathbf{\Delta}_{--}^{-}, 20 \%$.
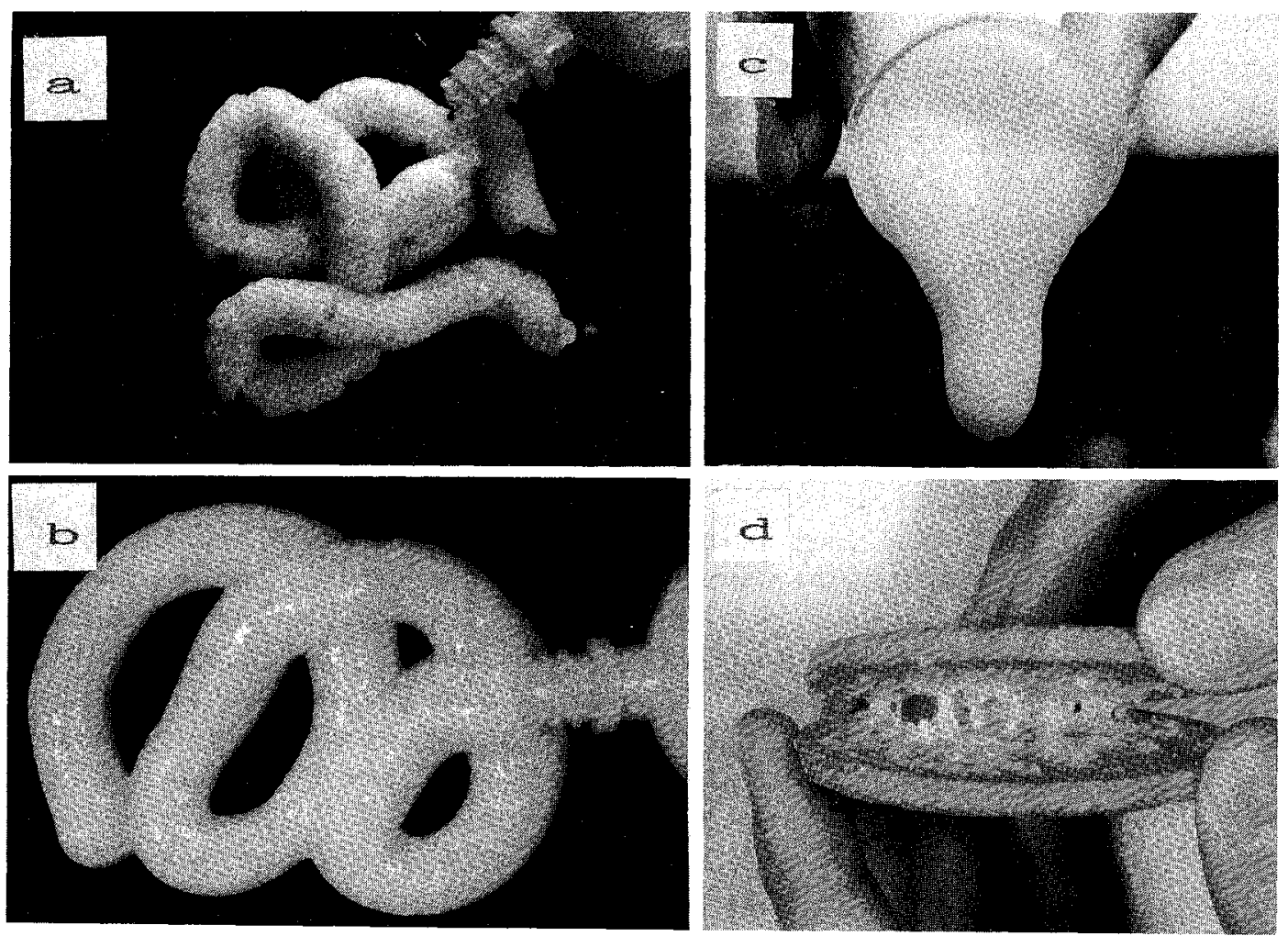

Fig. 6. Photographs of the Cooked Fish Paste.

a: Cooked fish paste supplemented with $150 \%$ of water and $4.5 \%$ of the pregelatinized corn starch treated by the extruder.

b: Cooked fish paste supplemented with $150 \%$ of water and $4.5 \%$ of the pregelatinized tapioca starch treated by the drum drier.

c: The same cooked fish paste as in b.

d: Cooked fish paste supplemented with $150 \%$ of water, $4.5 \%$ of the pregelatinized tapioca starch treated by the drum drier, and $20 \%$ of butter. 
the viscosity of the cooked fish paste with 10 and $20 \%$ of the salad oil or butter added apart from the $4.5 \%$ of pregelatinized potato or tapioca starch treated with the drum drier. The viscosity was remarkably decreased by raising the temperature, but conversely increased by increasing the amount of salad oil or butter added. Furthermore, the faintly remaining fishy odour of the cooked fish paste was nearly masked, and good body was given to the taste by their addition.

Figure 6 shows the photographs of the especially characteristic cooked fish paste prepared in the present study. Figures $6 \mathrm{a}$ and $6 \mathrm{~b}$ show the cooked fish paste squeezed out from a tube. A somewhat coarse texture could be felt for the cooked fish paste supplemented with $150 \%$ of water and $4.5 \%$ of corn starch treated with the extruder (Fig. 6a). On the contrary, a particularly fine texture was felt for the paste supplemented with $150 \%$ of water and $4.5 \%$ of the tapioca starch treated with the drum drier (Fig. 6b). The marked viscosity of the latter paste can be understood from Fig. 6c. Figure 6d shows the fish paste supplemented with $150 \%$ of water, $4.5 \%$ of the tapioca starch treated with the extruder, and $20 \%$ of butter. The very sticky features are apparent.

The viscous cooked fish pastes obtained in this way are quite new marine foods, although they are fundamentally classified into the usual cooked surimi product. The cooked fish pastes supplemented with 150 or $300 \%$ of water and $4.5-6.75 \%$ of the potato or tapioca starch were felt to the most agreeable in taste.

The other marked feature of these cooked fish pastes was that little protein coagulation due to acid-denaturation was induced even if rice vinegar was added, and that the mixture of the paste and vinegar was well miscible with salad oil or butter. Presumably, the cooked fish flesh would act as an emulsifier in the presence of the pregelatinized starch. For example, a dressing type of paste was obtained by grinding the paste supplemented with $150 \%$ of water, $4.5 \%$ of tapioca starch treated with the drum drier and $26 \%$ of rice vinegar (Nakano Vinegar Co., Ltd., Rice Vinegar) in a mortar for $5 \mathrm{~min}$, which was followed by grinding with $160 \%$ of salad oil for a further $5 \mathrm{~min}$. A tasty French dressing type of product would be given if adequate seasonings and condiments are added. In order to preserve such a product, however, the preventive measures for retrogradation of the starch should be studied.

\section{Reference}

1) M. Okada, Bull. Tokai Reg. Res. Lab., No. 36, 21 (1963). 